\title{
Increased Fluorodeoxyglucose Uptake Following Endovascular Abdominal Aortic Aneurysm Repair: A Predictor of Endoleak?
}

\author{
Kosmas I. Paraskevas ${ }^{1, *}$, Alexandros A. Tzovaras ${ }^{2}$, Vassilios Stathopoulos ${ }^{3}$, Fotini Gentimi ${ }^{4}$ and \\ Dimitri P. Mikhailidis 5 \\ ${ }^{I}$ Department of Vascular Surgery, Red Cross Hospital, Athens, Greece \\ ${ }^{2} 1^{\text {st }}$ Department of Oncology, Aghios Savvas Hospital, Athens, Greece \\ ${ }^{3} 2^{\text {nd }}$ Department of Surgery, Rhodes General Hospital, Rhodes, Greece \\ ${ }^{4} 2^{\text {nd }}$ Department of Pediatric Surgery, Aghia Sophia Children's Hospital, Athens, Greece \\ ${ }^{5}$ Department of Clinical Biochemistry (Vascular Disease Prevention Clinics), Royal Free Hospital Campus, University \\ College London Medical School, University College London (UCL), London, UK
}

\begin{abstract}
The main criterion for abdominal aortic aneurysm (AAA) repair is an AAA diameter $\geq 5.5 \mathrm{~cm}$. However, some AAAs rupture when they are smaller. Size alone may therefore not be a sufficient criterion to determine rupture risk. Fluorodeoxyglucose (FDG) uptake is increased in the presence of inflammation and it was suggested that this may be a better predictor of rupture risk than AAA size. Furthermore, increased FDG uptake following endovascular AAA repair may be an indirect predictor of continuous AAA sac enlargement due to the presence of an endoleak (even if this is not detected by imaging modalities) and/or increased AAA rupture risk. The role of FDG uptake needs to be explored further in the management of AAAs.
\end{abstract}

Keywords: Abdominal aortic aneurysm, fluorodeoxyglucose, endovascular aneurysm repair, rupture risk, predictor, endoleak.

\section{INTRODUCTION}

According to current guidelines [1] the main criterion for abdominal aortic aneurysm (AAA) repair is a diameter $\geq 5.5 \mathrm{~cm}$. However, smaller AAAs can rupture and AAAs are discovered after exceeding this diameter without rupturing $[2,3]$.

Fluorodeoxyglucose (FDG) uptake, measured by positron emission tomography (PET), is increased in the presence of inflammation [4]. In turn, AAAs are characterized by activation of inflammatory/immune cells causing degradation of elastin and collagen, destruction of medial elastic tissue, medial neovascularization and a decrease in vascular smooth muscle cells [5]. It follows that an association between FDG uptake by the AAA wall and the processes leading to rupture has been reported $[3,6,7]$.

Although FDG correlates with inflammation of the AAA wall and rupture risk $[3,6,7]$, it does not correlate with maximal AAA diameter [8]. Nevertheless, it was recently proposed that FDG may be a better predictor of AAA instability and rupture than AAA size [9]. Such an association holds implications for the management of AAAs [9]. FDG uptake may also predict endoleaks following endovascular

*Address correspondence to this author at the Department of Vascular Surgery, Red Cross Hospital, 24, Papagou street, Athens 141 22, Greece; Tel: +30 697777 6202; Fax: +30 2103215 792;

E-mail: paraskevask@hotmail.com
AAA repair (EVAR) even if this is not detected by imaging modalities. This article considers this hypothesis.

\section{INCREASED FDG UPTAKE AFTER EVAR: AN INDIRECT SIGN OF AN ENDOLEAK?}

The most common complication of EVAR is an endoleak which is the persistence of perigraft blood flow inside the AAA sac [10-13]. Endoleaks are associated with adverse outcomes, including AAA sac growth, the need for conversion to open repair, high reintervention rates and rupture [1013]. Therefore, following EVAR long-term (possibly lifelong) imaging surveillance is recommended [10-13].

Several imaging techniques for the surveillance of patients following EVAR have been described including plain radiography, ultrasound, computed tomography (CT)/CT angiography and magnetic resonance (MR) imaging/MR angiography $[13,14]$. Each technique has its advantages and disadvantages [13, 14]. Despite the availability of advanced imaging modalities some endoleaks may still be missed. Poor compliance with the follow-up schedule may be a reason but in some cases, the endoleak is missed due to technical deficiencies/shortcomings [12, 15-18].

A recent report systematically reviewing the pathogenesis, etiology and timing of AAA rupture following EVAR identified a total of 270 patients with AAA rupture after EVAR [12]. The cause of AAA rupture was known for 235 patients. Endoleaks were the main cause of rupture in 160 of 
the 235 patients (type IA: 57 patients; type IB: 31 patients; type II: 23 patients; type III: 26 patients; type IV: 0 patients; endotension: 9 patients). The endoleak type was not specified in 14 cases with rupture due to endoleak [12].

The presence of an endoleak at the last follow-up before rupture was described for only 56 of the 160 patients in whom the main cause of rupture was an endoleak [12]. A description of the course of AAA sac diameter during follow-up was presented in 101 patients. Enlargement of the AAA sac during follow-up occurred in 36 of these patients, no change was seen in 39 and shrinkage was reported in 26 patients. In 35 patients no abnormalities were found during follow-up (absence of endoleak, wire fractures, migration, graft angulation, insecure fixation, signs of inflammation and sac enlargement). In another 6 patients only a small type II endoleak was found during follow-up while the AAA sac was stable or shrunken. So, in 41 patients no abnormalities were found during follow-up that required re-intervention [12].

Another study evaluated the incidence and impact of previously unrecognized type II endoleaks using a modified intraoperative angiographic protocol (namely the use of digital subtraction fluoroscopy continuously for $60 \mathrm{sec}$ after injections of $20 \mathrm{ml}$ iodinated contrast both in the pararenal aorta and within the endovascular graft) [16]. A total of 391 patients undergoing EVAR were evaluated (standard completion angiograms: 264 patients; modified angiographic protocol: 127 patients). Type II endoleaks were detected intraoperatively in more patients in whom the modified compared with the standard angiographic protocol was used (53 of 127 vs 12 of 264 , or $41 \%$ vs $6 \%$, respectively; $\mathrm{p}<0.001)$ [16]. A third study aimed to analyze the clinical implications of endoleaks documented by CT angiography which were missed by color duplex ultrasound in 232 patients undergoing EVAR during a 5-year period [17]. All patients were followed by both CT angiography and color duplex ultrasound at 1 month following the procedure and every 6 months thereafter. A total of 39 endoleaks were detected using CT angiography compared with only 28 using ultrasonography. Overall, color duplex ultrasonography failed to identify an endoleak in $>25 \%$ of the cases (11 of 39 endoleaks [28\%]; 2 late type I, 6 early type II, 2 late type II and 1 early type IV) [17]. Finally, in a single-center report of 445 AAA patients treated endovascularly, late AAA rupture occurred in 3 cases [18]. In all cases, the reason for rupture was type I endoleak that was not diagnosed during postEVAR surveillance scans [18].

\section{COMMENT}

Despite a wide variety of imaging modalities, some endoleaks are missed [12, 15-18]. Ultrasonography is a costeffective and reproducible method that identifies an endoleak in the majority of the cases; nevertheless, it may miss as many as $28 \%$ of endoleaks [17]. Due to its costeffectiveness, ultrasonography should remain the primary diagnostic tool for detecting endoleaks following EVAR. When a follow-up ultrasound examination is negative, however, FDG uptake could represent an investigation which could alert the physician about the presence of an endoleak following EVAR. However, since most ultrasound examinations will be negative, the cost of an additional investigation by FDG uptake is prohibitive unless there is a high index of clinical suspicion of an endoleak. Moreover, the FDG uptake will not accurately reveal the location of the endoleak and AAA infection could lead to increased FDG uptake (due to local inflammation) and result in misinterpretation [19]. As FDG represents an inflammatory state [3-7], it would be interesting to explore the role of drugs possessing antiinflammatory action. Through inhibition/decrease of AAA expansion rates, statins may play a role in the management of AAAs [20]. Oxidative stress may play a role in the pathogenesis of AAAs; thus statins may exert their growth inhibitory effect by interfering with this pathway [21]. Irrespective of an effect on AAA growth, all AAA patients undergoing surgery [22] or a percutaneous intervention [23] should receive statin therapy to improve perioperative morbidity and mortality rates. Future studies should investigate the effect of statin therapy on post-EVAR patients exhibiting increased FDG uptake.

Apart from research applications, FDG uptake may prove useful in predicting the risk of rupture in AAA with a diameter below the threshold for intervention as assessed by ultrasound. This is of interest since there is a current debate as to whether there are grounds for intervening with EVAR in AAAs below the threshold diameter. Based on the lower perioperative mortality rates associated with EVAR compared with open surgical procedures [24], it was proposed that the current size threshold for elective AAA repair may need to be lowered in the endovascular era [25, 26]. Two ongoing multicentre randomized controlled trials, Comparison of surveillance versus Aortic Endografting for Small Aneurysm Repair (CAESAR) [25] in Europe and Positive Impact of endoVascular Options for Treating Aneurysms earLy [PIVOTAL] [26] in the United States are currently comparing EVAR vs surveillance for AAAs $<5 \mathrm{~cm}$ in size. This argument would be supported even further if a reliable predictor (i.e. FDG uptake) of expansion and possible eventual rupture risk was available. This option requires further consideration.

\section{REFERENCES}

[1] Hirsch AT, Haskal ZJ, Hertzer NR, et al; American Association for Vascular Surgery; Society for Vascular Surgery; Society for Cardiovascular Angiography and Interventions; Society for Vascular Medicine and Biology; Society of Interventional Radiology; ACC/AHA Task Force on Practice Guidelines Writing Committee to Develop Guidelines for the Management of Patients With Peripheral Arterial Disease; American Association of Cardiovascular and Pulmonary Rehabilitation; National Heart, Lung, and Blood Institute; Society for Vascular Nursing; TransAtlantic Inter-Society Consensus; Vascular Disease Foundation. ACC/AHA 2005 Practice Guidelines for the management of patients with peripheral arterial disease (lower extremity, renal, mesenteric, and abdominal aortic): a collaborative report from the American Association for Vascular Surgery/Society for Vascular Surgery, Society for Cardiovascular Angiography and Interventions, Society for Vascular Medicine and Biology, Society of Interventional Radiology, and the ACC/AHA Task Force on Practice Guidelines (Writing Committee to Develop Guidelines for the Management of Patients With Peripheral Arterial Disease): endorsed by the American Association of Cardiovascular and Pulmonary Rehabilitation; National Heart, Lung, and Blood Institute; Society for Vascular Nursing; TransAtlantic Inter-Society Consensus; and Vascular Disease Foundation. Circulation 2006; 113: e463-654.

[2] Fillinger M. Who should we operate on and how do we decide: predicting rupture risk and survival in patients with aortic aneurysm. Semin Vasc Surg 2007; 20: 121-7. 
[3] Reeps C, Essler M, Pelisek J, Seidl S, Eckstein HH, Krause BJ. Increased 18F-fluorodeoxyglucose uptake in abdominal aortic aneurysms in positron emission/computed tomography is associated with inflammation, aortic wall instability, and acute symptoms. J Vasc Surg 2008; 48: 417-23.

[4] Tawakol A, Migrino RQ, Hoffmann U, et al. Noninvasive in vivo measurement of vascular inflammation with F-18 fluorodeoxyglucose positron emission tomography. J Nucl Cardiol 2005; 12: 294301.

[5] Shimizu K, Mitchell RN, Libby P. Inflammation and cellular immune response in abdominal aortic aneurysms. Arterioscler Thromb Vasc Biol 2006; 26: 987-94.

[6] Sakalihasan N, Van Damme H, Gomez P, et al. Positron emission tomography (PET) evaluation of abdominal aortic aneurysm (AAA). Eur J Vasc Endovasc Surg 2002; 23: 431-6.

[7] Sakalihasan N, Hustinx R, Limet R. Contribution of PET scanning to the evaluation of abdominal aortic aneurysm. Semin Vasc Surg 2004; 17: 144-53.

[8] Truijers M, Kurvers HA, Bredie SJ, Oyen WJ, Blankensteijn JD. In vivo imaging of abdominal aortic aneurysms: increased FDG uptake suggests inflammation in the aneurysm wall. J Endovasc Ther 2008; 15: 462-7.

[9] Paraskevas KI. Regarding "Increased 18F-fluorodeoxyglucose uptake in abdominal aortic aneurysms in positron emission/computed tomography is associated with inflammation, aortic wall instability, and acute symptoms". J Vasc Surg 2009; 49: 1631.

[10] Harris PL, Vallabhaneni SR, Desgranges P, Becquemin JP, van Marrewijk C, Laheij RJ. Incidence and risk factors of late rupture, conversion, and death after endovascular repair of infrarenal aortic aneurysms: the EUROSTAR experience. European Collaborators on Stent/graft techniques for aortic aneurysm repair. J Vasc Surg 2000; 32: 739-49.

[11] Bernhard VM, Mitchell RS, Matsumura JS, et al. Ruptured abdominal aortic aneurysm after endovascular repair. J Vasc Surg 2002; 35: 1155-62.

[12] Schlosser FJ, Gusberg RJ, Dardik A, et al. Aneurysm rupture after EVAR: can the ultimate failure be predicted? Eur J Vasc Endovasc Surg 2009; 37: 15-22.

[13] Stavropoulos SW, Charagundla SR. Imaging techniques and management of endoleaks after endovascular aortic aneurysm repair. Radiology 2007; 243: 641-55.

[14] Kranokpiraksa P, Kaufman JA. Follow-up of endovascular aneurysm repair: plain radiography, ultrasound, CT/CT angiography, MR imaging/MR angiography, or what? J Vasc Interv Radiol 2008; 19: S27-36.
[15] Fransen GA, Vallabhaneni SR Sr, van Marrewijk CJ, Laheij RJ, Harris PL, Buth J; EUROSTAR. Rupture of infra-renal aortic aneurysm after endovascular repair: a series from EUROSTAR registry. Eur J Vasc Endovasc Surg 2003; 26: 487-93.

[16] Faries PL, Briggs VL, Bernheim J, Kent KC, Hollier LH, Marin ML. Increased recognition of type II endoleaks using a modified intraoperative angiographic protocol: implications for intermittent endoleak and aneurysm expansion. Ann Vasc Surg 2003; 17: 60814.

[17] AbuRahma AF. Fate of endoleaks detected by CT angiography and missed by color duplex ultrasound in endovascular grafts for abdominal aortic aneurysms. J Endovasc Ther 2006; 13: 490-5.

[18] Szmidt J, Galazka Z, Rowinski O, et al. Late aneurysm rupture after endovascular abdominal aneurysm repair. Interact Cardiovasc Thorac Surg 2007; 6: 490-4.

[19] Zimmerman PM, Cherr GS, Angelo GC, Gona J, Dosluoglu HH. Is F 18 fluorodeoxyglucose positron emission tomography too sensitive for the diagnosis of vascular endograft infection? Vascular 2008; 16: 346-9.

[20] Paraskevas KI, Liapis CD, Hamilton G, Mikhailidis DP. Are statins an option in the management of abdominal aortic aneurysms? Vasc Endovascular Surg 2008; 42: 128-34.

[21] Paraskevas KI, Andrikopoulou M, Anastasakis E, Perrea D, Mikhailidis DP. Oxidative stress in the pathogenesis of abdominal aortic aneurysms: a possible pathway for the effect of statins? Angiology 2010; 61: 226-7.

[22] Paraskevas KI, Liapis CD, Hamilton G, Mikhailidis DP. Can statins reduce perioperative morbidity and mortality in patients undergoing non-cardiac vascular surgery? Eur J Vasc Endovasc Surg 2006; 32: 286-93.

[23] Paraskevas KI, Athyros VG, Briana DD, Kakafika AI, Karagiannis A, Mikhailidis DP. Statins exert multiple beneficial effects on patients undergoing percutaneous revascularization procedures. Curr Drug Targets 2007; 8: 942-51.

[24] Schermerhorn ML, O'Malley AJ, Jhaveri A, Cotterill P, Pomposelli F, Landon BE. Endovascular vs. open repair of abdominal aortic aneurysms in the Medicare population. N Engl J Med 2008; 358: 464-74.

[25] Cao P. CAESAR Trial Collaborators. Comparison of surveillance vs Aortic Endografting for Small Aneurysm Repair (CAESAR) trial: study design and progress. Eur J Vasc Endovasc Surg 2005; 30: $245-51$

[26] Ouriel K. Treating smaller AAAs. Does the availability of endovascular grafts change the size threshold for repair? Endovascular Today 2005; 3: 39-42. Available at: http://www.evtoday.com/PDF articles/0305/F1_Ouriel.html. 